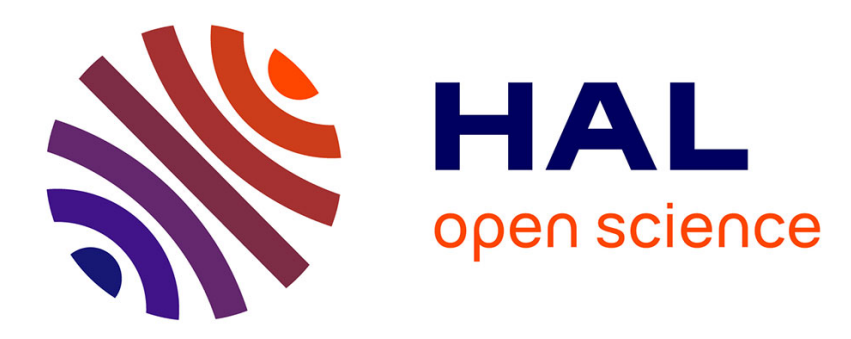

\title{
Correction of some formulas of Agarwal's fast Fourier transform least-squares algorithm
}

\author{
Alain Lifchitz
}

\section{To cite this version:}

Alain Lifchitz. Correction of some formulas of Agarwal's fast Fourier transform least-squares algorithm. Acta Crystallographica Section A : Foundations and Advances [2014-..], 1986, 42, pp.204. 10.1107/S0108767386099518. hal-00015164

\section{HAL Id: hal-00015164 \\ https://hal.science/hal-00015164}

Submitted on 23 Feb 2006

HAL is a multi-disciplinary open access archive for the deposit and dissemination of scientific research documents, whether they are published or not. The documents may come from teaching and research institutions in France or abroad, or from public or private research centers.
L'archive ouverte pluridisciplinaire HAL, est destinée au dépôt et à la diffusion de documents scientifiques de niveau recherche, publiés ou non, émanant des établissements d'enseignement et de recherche français ou étrangers, des laboratoires publics ou privés. 
Acta Cryst. (1986). A42, 204

Correction of some formulas of Agarwal's fast Fourier transform least-squares algorithm. By ALAIN LIfChITz, Laboratoire de Minéralogie et de Cristallographie, associé au CNRS, Université Pierre et Marie Curie (Paris VI) et Paris VII, 4 place Jussieu, F-75230 Paris CEDEX 05, France

(Received 5 November 1985; accepted 3 February 1986)

\section{Abstract}

The formulas (34), (37) and (38) of Agarwal [Acta Cryst. (1978), A34, 791-809] should be changed to:

$$
\begin{aligned}
H_{2}\left(B_{m}, B_{n}\right)= & \sum_{\mathbf{s}}+\frac{1}{2} g_{m}(\mathbf{s}) g_{n}(\mathbf{s})\left(s^{4} / 16\right) W(\mathbf{s}) \\
& \times \exp [i 2 \varphi(\mathbf{s})] \exp \left[-i 2 \pi \mathbf{s} .\left(\mathbf{r}_{m}+\mathbf{r}_{n}\right)\right]
\end{aligned}
$$

$H_{1}\left(x_{m}, B_{n}\right)=\sum_{s}-\frac{1}{4} g_{m}(\mathbf{s}) g_{n}(\mathrm{~s})\left(i \pi h s^{2}\right) W(\mathrm{~s})$

$$
\begin{aligned}
& \times \exp \left[+i 2 \pi \mathbf{s} \cdot\left(\mathbf{r}_{m}-\mathbf{r}_{n}\right)\right] \\
H_{2}\left(x_{m}, B_{n}\right)= & \sum_{\mathbf{s}}+\frac{1}{4} g_{m}(\mathbf{s}) g_{n}(\mathbf{s})\left(i \pi h s^{2}\right) W(\mathbf{s}) \\
& \times \exp [i 2 \varphi(\mathbf{s})] \exp \left[-i 2 \pi \mathbf{s} \cdot\left(\mathbf{r}_{m}+\mathbf{r}_{n}\right)\right] .
\end{aligned}
$$

All the information is contained in the Abstract. 